\title{
Properties enhancement using oil palm shell nanoparticles of fibers reinforced polyester hybrid composites
}

\begin{abstract}
Oil palm shell (OPS) nanoparticles were utilized as filler in fibers reinforced polyester hybrid composites. The OPS nanoparticles were successfully produced from the raw OPS using high-energy ball milling process. Fundamental properties including morphology, crystalline size, and particle size of the OPS nanoparticles were determined. Tri-layer natural fiber reinforcement (kenafi coconutï kenaf fiber mat) polyester hybrid composites were prepared by hand lay-up techniques. The influences of the OPS nanoparticles loading in the natural fibers reinforced polyester hybrid composites were determined by analyzing physical, mechanical, morphological, and thermal properties of the composites. Results showed that the incorporation of the OPS nanoparticles into the hybrid composites enhanced the composite properties. Further, the natural fibers reinforced polyester hybrid composite had the highest physical, mechanical, morphological, and thermal characteristics at $3 \mathrm{wt} \%$ OPS nanoparticles loading.
\end{abstract}

Keyword: Hybrid composite; Natural fiber; Polymerï matrix composites; Mechanical properties; Physical properties 